\title{
STIMULATED RAMAN SCATTERING OF NONCLASSICAL LIG̈HT BY SQUEEZED PHONONS
}

\author{
J. PER̆ina, M. KÁRSKÁ, AND J. KřEPELKA \\ Department of Optics, Palacký University \\ Tr̃. Svobody 26, 77146 Olomouc, Czechoslovakia
}

(Received September 1990; in revised version March 19, 1991)

\begin{abstract}
Quantum statistical properties are derived for stimulated Raman scattering by squeezed phonons including squeezing of vacuum fluctuations in radiation modes and sub-Poisson photon behaviour. Initial radiation can be in squeezed thermalized and/or sub-Poisson states, phonon system can also be squeezed. In particular, effects of initial phase conditions, losses, initial nonclassical behaviour and phonon squeezing are demonstrated in terms of reduced factorial moments up to the fifth order in combined photon-photon and photon-phonon modes. Also photon number distribution, quadrature variances and principal squeeze parameters are shown.
\end{abstract}

PACS numbers: 42.50

\section{Introduction}

It is possible to develop a quantum description of Brillouin and Raman scattering including photon statistics and correlation properties of photons and phonons using various approaches in the framework of both the Heisenberg and Schrödinger pictures (see e.g. [1-4] and references therein; a review can be found in [5]). In this paper we consider stimulated Raman scattering of squeezed and/or sub-Poisson light with additional noise components by squeezed phonons. The laser field is assumed to be so strong that it can be described classically, although a similar treatment involving laser mode depletion can also be developed. The phonon system is effectively described by a single mode which can be squeezed. Making use of the reduced factorial moments, in some cases up to the fifth order, and photon number distributions, we discuss sub-Poisson regimes of scattering. Adopting quadrature variances and principal squeeze parameters, we can find regimes for 
squeezing of vacuum fluctuations in radiation modes provided that the initial radiation field is squeezed and/or sub-Poissonian. Losses and various initial phase conditions are included.

In Sec. 2 we describe the basic model of nonlinear dynamics including time development of quantum characteristic function and evolution of basic quantum statistical characteristics, such as photon number distribution , principal squeeze $^{-}$ parameter, etc., whereas in Sec. 3 the results obtained are discussed and demonstrated.

\section{Quantum dynamics}

The photon-photon interaction for the process under discussion can be described by the effective Hamiltonian

$$
\hat{H}=\hat{H}_{0}+\hat{H}_{i}+\hat{H}_{\text {add }}
$$

where

$$
\hat{H}_{0}=\hbar \omega_{\mathrm{S}}\left(\hat{a}_{\mathrm{S}}^{\dagger} \hat{a}_{\mathrm{S}}+\frac{1}{2}\right)+\hbar \omega_{\mathrm{A}}\left(\hat{a}_{\mathrm{A}}^{\dagger} \hat{a}_{\mathrm{A}}+\frac{1}{2}\right)+\hbar \omega_{\mathrm{V}}\left(\hat{a}_{\mathrm{V}}^{\dagger} \hat{a}_{\mathrm{V}}+\frac{1}{2}\right)
$$

is the free-field Hamiltonian for Stokes (S), anti-Stokes (A), and vibration phonons (V) modes, respectively, $\hat{a}_{\mathrm{S}}\left(\hat{a}_{\mathrm{S}}^{\dagger}\right), \hat{a}_{\mathrm{A}}\left(\hat{a}_{\mathrm{A}}^{\dagger}\right), \hat{a}_{\mathrm{V}}\left(\hat{a}_{\mathrm{V}}^{\dagger}\right)$ being the annihilation (creation) operators of these modes with frequencies $\omega_{\mathrm{S}}, \omega_{\mathrm{A}}$, and $\omega_{\mathrm{V}}$; assuming pumped laser light with the frequency $\omega_{\mathrm{L}}$ and phase $\varphi_{\mathrm{L}}$ to be coherent and so strong that it can be described classically, we can write down the interaction Hamiltonian in the form [1]

$$
\hat{H}_{i}=-\left[\hbar g_{\mathrm{S}} \hat{a}_{\mathrm{S}}^{\dagger} \hat{a}_{\mathrm{V}}^{\dagger} \exp \left(-\mathrm{i} \omega_{\mathrm{L}} t+\mathrm{i} \varphi_{\mathrm{L}}\right)+\hbar g_{\mathrm{A}} \hat{a}_{\mathrm{V}} \hat{a}_{\mathrm{A}}^{\dagger} \exp \left(-\mathrm{i} \omega_{\mathrm{L}} t+\mathrm{i} \varphi_{\mathrm{L}}\right)+\text { h.c. }\right]
$$

here $g_{\mathrm{S}}$ and $g_{\mathrm{A}}$ are the Stokes and anti-Stokes coupling constants, respectively and h.c. means the hermitian conjugate terms. The interaction of radiative and phonon modes with reservoirs describes -correctly losses in the modes and it is specified by the additional Hamiltonian

$$
\hat{H}_{\mathrm{add}}=\hbar \sum_{j=\mathrm{S}, \mathrm{A}, \mathrm{V}} \sum_{l}\left[\psi_{l}^{(j)}\left(\hat{b}_{l}^{(j) \dagger} \hat{b}_{l}^{(j)}+\frac{1}{2}\right)+\kappa_{j l} \hat{a}_{\mathrm{j}}^{\dagger} \hat{b}_{l}^{(j)}+\kappa_{j l}^{*} \hat{a}_{\mathrm{j}} \hat{b}_{l}^{(j) \dagger}\right]
$$

where $\psi_{l}^{(j)}$ are the reservoir frequencies $\left(\psi_{l}^{(j)} \approx \omega_{j}, j=\mathrm{S}, \mathrm{A}, \mathrm{V}\right), \kappa_{j l}$ are the coupling constants of photon and phonon modes with their reservoirs, and $\hat{b}_{l}^{(j)}\left(\hat{b}_{l}^{(j) \dagger}\right)$ are the corresponding annihilation (creation) reservoir operators. All these operators obey the standard boson commutation rules

$$
\left[\hat{a}_{j}, \hat{a}_{k}^{\dagger}\right]=\delta_{j k}, \quad\left[\hat{b}_{l}^{(j)}, \hat{b}_{m}^{(k) \dagger}\right]=\delta_{l m} \delta_{j k}, \quad j, k=\mathrm{S}, \mathrm{A}, \mathrm{V}
$$


with all other commutators zero. The frequency resonance conditions are

$$
\omega_{\mathrm{S}}=\omega_{\mathrm{L}}-\omega_{\mathrm{V}}, \quad \omega_{\mathrm{A}}=\omega_{\mathrm{L}}+\omega_{\mathrm{V}} .
$$
The corresponding Heisenberg equations have been solved in $[1,2]$ providing
for their solution

$$
\begin{aligned}
& \hat{a}_{\mathrm{S}}(t)=\mu_{\mathrm{S}}(t) \hat{a}_{\mathrm{S}}+\mu_{\mathrm{A}}(t) \hat{a}_{\mathrm{A}}^{\dagger}+\mu_{\mathrm{V}}(t) \hat{a}_{\mathrm{V}}^{\dagger}+\hat{L}_{\mathrm{S}}(t), \\
& \hat{a}_{\mathrm{A}}(t)=\nu_{\mathrm{A}}(t) \hat{a}_{\mathrm{A}}+\nu_{\mathrm{S}}(t) \hat{a}_{\mathrm{S}}^{\dagger}+\nu_{\mathrm{V}}(t) \hat{a}_{\mathrm{V}}+\hat{L}_{\mathrm{A}}(t), \\
& \hat{a}_{\mathrm{V}}(t)=\lambda_{\mathrm{V}}(t) \hat{a}_{\mathrm{V}}+\lambda_{\mathrm{S}}(t) \hat{a}_{\mathrm{S}}^{\dagger}+\lambda_{\mathrm{A}}(t) \hat{a}_{\mathrm{A}}+\hat{L}_{\mathrm{V}}(t),
\end{aligned}
$$

where $\hat{L}_{\mathrm{S}, \mathrm{A}, \mathrm{V}}$ are Markovian Langevin forces $[1], \hat{a}_{\mathrm{j}} \equiv \hat{a}_{\mathrm{j}}(0)$ and

$$
\begin{gathered}
\mu_{\mathrm{S}}(t)=\left[1+\frac{\left|g_{\mathrm{S}}\right|^{2}}{\Omega^{2}}(1-\cos \Omega t)\right] \exp (-\gamma t / 2), \\
\mu_{\mathrm{A}}(t)=-\nu_{\mathrm{S}}(t)=\frac{g_{\mathrm{S}} g_{\mathrm{A}}}{\Omega^{2}}(1-\cos \Omega t) \exp \left(2 \mathrm{i} \varphi_{\mathrm{L}}-\gamma t / 2\right), \\
\mu_{\mathrm{V}}(t)=\frac{\mathrm{i} g_{\mathrm{S}}}{\Omega^{2}} \sin \Omega t \exp \left(\mathrm{i} \varphi_{\mathrm{L}}-\gamma t / 2\right), \\
\nu_{\mathrm{A}}(t)=\left[1-\frac{\left|g_{\mathrm{A}}\right|^{2}}{\Omega^{2}}(1-\cos \Omega t)\right] \exp (-\gamma t / 2), \\
\nu_{\mathrm{V}}(t) \doteq \frac{\mathrm{i} g_{\mathrm{A}}}{\Omega^{2}} \sin \Omega t \exp \left(\mathrm{i} \varphi_{\mathrm{L}}-\gamma t / 2\right), \\
\lambda_{\mathrm{S}}(t)=\frac{\mathrm{i} g_{\mathrm{S}}}{\Omega^{2}} \sin \Omega t \exp \left(\mathrm{i} \varphi_{\mathrm{L}}-\gamma t / 2\right), \\
\lambda_{\mathrm{A}}(t)=\frac{\mathrm{i} g_{\mathrm{A}}^{*}}{\Omega^{2}} \sin \Omega t \exp \left(\mathrm{i} \varphi_{\mathrm{L}}-\gamma t / 2\right), \\
\lambda_{\mathrm{V}}(t)=\cos \Omega t \exp (-\gamma t / 2), \\
\Omega^{2}=\left|g_{\mathrm{A}}\right|^{2}-\left|g_{\mathrm{S}}\right|^{2} ;
\end{gathered}
$$

here $\gamma=\gamma_{\mathrm{S}}=\gamma_{\mathrm{A}}=\gamma_{\mathrm{V}}$ are the damping constants.

The corresponding antinormal characteristic function with parameters $\beta_{j}$ can be written in the form [6-9]

$$
\begin{gathered}
C_{\mathrm{A}}\left(\left\{\beta_{j}\right\}, t\right)=\exp \left\{\sum _ { j = \mathrm { S } , \mathrm { A } , \mathrm { V } } \left[\left(\beta_{j} \xi_{j}^{*}(t)-\beta_{j}^{*} \xi_{j}(t)\right)-B_{j}(t)\left|\beta_{j}\right|^{2}\right.\right. \\
\left.\left.+\frac{1}{2}\left(C_{j}(t) \beta_{j}^{* 2}+\text { c.c. }\right)\right]+\sum_{j<k}\left[\left(D_{j k}(t) \beta_{j}^{*} \beta_{k}^{*}+\bar{D}_{j k}(t) \beta_{j} \beta_{k}^{*}\right)+\text { c.c. }\right]\right\},
\end{gathered}
$$


where the set $(\mathrm{S}, \mathrm{A}, \mathrm{V})$ is assumed to be ordered, complex field amplitudes $\xi_{j}(t)$ are determined by

$$
\begin{aligned}
& \xi_{\mathrm{S}}(t)=\mu_{\mathrm{S}}(t) \xi_{\mathrm{S}}+\mu_{\mathrm{A}}(t) \xi_{\mathrm{A}}^{*}+\mu_{\mathrm{V}}(t) \xi_{\mathrm{V}}^{*} \\
& \xi_{\mathrm{A}}(t)=\nu_{\mathrm{S}}(t) \xi_{\mathrm{S}}^{*}+\nu_{\mathrm{A}}(t) \xi_{\mathrm{A}}+\nu_{\mathrm{V}}(t) \xi_{\mathrm{V}} \\
& \xi_{\mathrm{V}}(t)=\lambda_{\mathrm{S}}(t) \xi_{\mathrm{S}}^{*}+\lambda_{\mathrm{A}}(t) \xi_{\mathrm{A}}+\lambda_{\mathrm{V}}(t) \xi_{\mathrm{V}}
\end{aligned}
$$

$\xi_{j} \equiv \xi_{j}(0)$ being the initial complex amplitudes and the quantum noise functions $B_{j}(t), C_{j}(t), D_{j k}(t)$ and $\bar{D}_{j k}(t)(j \neq k)$ are given in the interaction picture $\left(\hat{a}_{\mathrm{j}}(t)=\hat{A}_{\mathrm{j}}(t) \exp \left(-\mathrm{i} \omega_{j} t\right)\right)$ by

$$
\begin{gathered}
C_{j}(t)=\left\langle\left(\Delta \hat{A}_{j}(t)\right)^{2}\right\rangle, \\
B_{j}(t)=\left\langle\Delta \hat{A}_{j}(t) \Delta \hat{A}_{j}^{\dagger}(t)\right\rangle=B_{j}^{*}(t), \\
D_{i j}(t)=\left\langle\Delta \hat{A}_{i}(t) \Delta \hat{A}_{j}(t)\right\rangle=D_{j i}(t), \\
\bar{D}_{i j}(t)=-\left\langle\Delta \hat{A}_{i}^{\dagger}(t) \Delta \hat{A}_{j}(t)\right\rangle=\bar{D}_{j i}^{*}(t) .
\end{gathered}
$$

Substituting solutions (2.5) in (2.8) one can find

$$
\begin{gathered}
C_{\mathrm{S}}(t)=\mu_{\mathrm{S}}^{2}(t) C_{\mathrm{S}}+\mu_{\mathrm{A}}^{2}(t) C_{\mathrm{A}}^{*}+\mu_{\mathrm{V}}^{2}(t) C_{\mathrm{V}}^{*} \\
C_{\mathrm{A}}(t)=\nu_{\mathrm{S}}^{2}(t) C_{\mathrm{S}}^{*}+\nu_{\mathrm{A}}^{2}(t) C_{\mathrm{A}}+\nu_{\mathrm{V}}^{2}(t) C_{\mathrm{V}} \\
C_{\mathrm{V}}(t)=\lambda_{\mathrm{S}}^{2}(t) C_{\mathrm{S}}^{*}+\lambda_{\mathrm{A}}^{2}(t) C_{\mathrm{A}}+\lambda_{\mathrm{V}}^{2}(t) C_{\mathrm{V}} \\
D_{\mathrm{VA}}(t)=\lambda_{\mathrm{S}}(t) \nu_{\mathrm{S}}(t) C_{\mathrm{S}}^{*}+\lambda_{\mathrm{A}}(t) \nu_{\mathrm{A}}(t) C_{\mathrm{A}}+\lambda_{\mathrm{V}}(t) \nu_{\mathrm{V}}(t) C_{\mathrm{V}} \\
\bar{D}_{\mathrm{VS}}(t)=-\lambda_{\mathrm{S}}^{*}(t) \mu_{\mathrm{S}}(t) C_{\mathrm{S}}-\lambda_{\mathrm{A}}^{*}(t) \mu_{\mathrm{A}}(t) C_{\mathrm{A}}^{*}-\lambda_{\mathrm{V}}^{*}(t) \mu_{\mathrm{V}}(t) C_{\mathrm{V}}^{*} \\
\bar{D}_{\mathrm{SA}}(t)=-\mu_{\mathrm{S}}^{*}(t) \nu_{\mathrm{S}}(t) C_{\mathrm{S}}^{*}-\mu_{\mathrm{A}}^{*}(t) \nu_{\mathrm{A}}(t) C_{\mathrm{A}}-\mu_{\mathrm{V}}^{*}(t) \nu_{\mathrm{V}}(t) C_{\mathrm{V}}
\end{gathered}
$$

where $C_{j} \equiv C_{j}(0)$; in these expressions there are no reservoir contributions. Similarly one obtains

$$
\begin{gathered}
B_{\mathrm{S}}(t)=\left|\mu_{\mathrm{S}}(t)\right|^{2} B_{\mathrm{S}}+\left|\mu_{\mathrm{A}}(t)\right|^{2}\left(B_{\mathrm{A}}-1\right)+\left|\mu_{\mathrm{V}}(t)\right|^{2}\left(B_{\mathrm{V}}-1\right) \\
+\gamma\left(\left\langle n_{\mathrm{dS}}\right\rangle+1\right) \int_{0}^{t}\left|\mu_{\mathrm{S}}\left(t^{\prime}\right)\right|^{2} \mathrm{~d} t^{\prime}+\gamma\left\langle n_{\mathrm{dA}}\right\rangle \int_{0}^{t}\left|\mu_{\mathrm{A}}\left(t^{\prime}\right)\right|^{2} \mathrm{~d} t^{\prime} \\
+\gamma\left\langle n_{\mathrm{dV}}\right\rangle \int_{0}^{t}\left|\mu_{\mathrm{V}}\left(t^{\prime}\right)\right|^{2} \mathrm{~d} t^{\prime} \\
B_{\mathrm{A}}(t)=\left|\nu_{\mathrm{S}}(t)\right|^{2}\left(B_{\mathrm{S}}-1\right)+\left|\nu_{\mathrm{A}}(t)\right|^{2} B_{\mathrm{A}}+\left|\nu_{\mathrm{V}}(t)\right|^{2} B_{\mathrm{V}} \\
+\gamma\left\langle n_{\mathrm{dS}}\right\rangle \int_{0}^{t}\left|\nu_{\mathrm{S}}\left(t^{\prime}\right)\right|^{2} \mathrm{~d} t^{\prime}+\gamma\left(\left\langle n_{\mathrm{dA}}\right\rangle+1\right) \int_{0}^{t}\left|\nu_{\mathrm{A}}\left(t^{\prime}\right)\right|^{2} \mathrm{~d} t^{\prime} \\
+\gamma\left(\left\langle n_{\mathrm{dV}}\right\rangle+1\right) \int_{0}^{t}\left|\nu_{\mathrm{V}}\left(t^{\prime}\right)\right|^{2} \mathrm{~d} t^{\prime}
\end{gathered}
$$




$$
\begin{gathered}
B_{\mathrm{V}}(t)=\left|\lambda_{\mathrm{S}}(t)\right|^{2}\left(B_{\mathrm{S}}-1\right)+\left|\lambda_{\mathrm{A}}(t)\right|^{2} B_{\mathrm{A}}+\left|\lambda_{\mathrm{V}}(t)\right|^{2} B_{\mathrm{V}} \\
+\gamma\left\langle n_{\mathrm{dS}}\right\rangle \int_{0}^{t}\left|\lambda_{\mathrm{S}}\left(t^{\prime}\right)\right|^{2} \mathrm{~d} t^{\prime}+\gamma\left(\left\langle n_{\mathrm{dA}}\right\rangle+1\right) \int_{0}^{t}\left|\lambda_{\mathrm{A}}\left(t^{\prime}\right)\right|^{2} \mathrm{~d} t^{\prime} \\
+\gamma\left(\left\langle n_{\mathrm{dV}}\right\rangle+1\right) \int_{0}^{t}\left|\lambda_{\mathrm{V}}\left(t^{\prime}\right)\right|^{2} \mathrm{~d} t^{\prime} \\
D_{\mathrm{VS}}(t)=\lambda_{\mathrm{S}}(t) \mu_{\mathrm{S}}(t)\left(B_{\mathrm{S}}-1\right)+\lambda_{\mathrm{A}}(t) \mu_{\mathrm{A}}(t) B_{\mathrm{A}}+\lambda_{\mathrm{V}}(t) \mu_{\mathrm{V}}(t) B_{\mathrm{V}} \\
+\gamma\left\langle n_{\mathrm{dS}}\right\rangle \int_{0}^{t} \lambda_{\mathrm{S}}\left(t^{\prime}\right) \mu_{\mathrm{S}}\left(t^{\prime}\right) \mathrm{d} t^{\prime}+\gamma\left(\left\langle n_{\mathrm{dA}}\right\rangle+1\right) \int_{0}^{t} \lambda_{\mathrm{A}}\left(t^{\prime}\right) \mu_{\mathrm{A}}\left(t^{\prime}\right) \mathrm{d} t^{\prime} \\
+\gamma\left(\left\langle n_{\mathrm{dV}}\right\rangle+1\right) \int_{0}^{t} \lambda_{\mathrm{V}}\left(t^{\prime}\right) \mu_{\mathrm{V}}\left(t^{\prime}\right) \mathrm{d} t^{\prime} \\
D_{\mathrm{SA}}(t)=\mu_{\mathrm{S}}(t) \nu_{\mathrm{S}}(t) B_{\mathrm{S}}+\mu_{\mathrm{A}}(t) \nu_{\mathrm{A}}(t)\left(B_{\mathrm{A}}-1\right)+\mu_{\mathrm{V}}(t) \nu_{\mathrm{V}}(t)\left(B_{\mathrm{V}}-1\right) \\
+\gamma\left(\left\langle n_{\mathrm{dS}}\right\rangle+1\right) \int_{0}^{t} \mu_{\mathrm{S}}\left(t^{\prime}\right) \nu_{\mathrm{S}}\left(t^{\prime}\right) \mathrm{d} t^{\prime}+\gamma\left\langle n_{\mathrm{dA}}\right\rangle \int_{0}^{t} \mu_{\mathrm{A}}\left(t^{\prime}\right) \nu_{\mathrm{A}}\left(t^{\prime}\right) \mathrm{d} t^{\prime} \\
\left.+\gamma\left\langle n_{\mathrm{dV}}\right\rangle\right) \int_{0}^{t} \mu_{\mathrm{V}}\left(t^{\prime}\right) \nu_{\mathrm{V}}\left(t^{\prime}\right) \mathrm{d} t^{\prime} \\
\bar{D}_{\mathrm{VA}}(t)=-\lambda_{\mathrm{S}}^{*}(t) \nu_{\mathrm{S}}(t) B_{\mathrm{S}}-\lambda_{\mathrm{A}}^{*}(t) \nu_{\mathrm{A}}(t)\left(B_{\mathrm{A}}-1\right)-\lambda_{\mathrm{V}}^{*}(t) \nu_{\mathrm{V}}(t)\left(B_{\mathrm{V}}-1\right) \\
+\gamma\left(\left\langle n_{\mathrm{dS}}\right\rangle+1\right) \int_{0}^{t} \lambda_{\mathrm{S}}^{*}\left(t^{\prime}\right) \nu_{\mathrm{S}}\left(t^{\prime}\right) \mathrm{d} t^{\prime}+\gamma\left\langle n_{\mathrm{dA}}\right\rangle \int_{0}^{t} \lambda_{\mathrm{A}}^{*}\left(t^{\prime}\right) \nu_{\mathrm{A}}\left(t^{\prime}\right) \mathrm{d} t^{\prime} \\
+\gamma\left\langle n_{\mathrm{dV}}\right\rangle \int_{0}^{t} \lambda_{\mathrm{V}}^{*}\left(t^{\prime}\right) \nu_{\mathrm{V}}\left(t^{\prime}\right) \mathrm{d} t^{\prime}
\end{gathered}
$$

where $B_{j} \equiv B_{j}(0)$ and there are reservoir contributions with mean numbers of reservoir oscillators $\left\langle n_{\mathrm{d} j}\right\rangle$ (for a way to include them see [9]). It is evident that in this way also initial coupling of modes could be included if we conserved the coefficients $D_{i j}(0), \bar{D}_{i j}(0) \neq 0$.

In general we assume initially squeezed light with additional noise components $\bar{n}_{j}$ so that

$$
B_{j}=\cosh ^{2}\left(r_{j}\right)+\bar{n}_{j}, \quad C_{j}=\frac{1}{2} \sinh \left(2 r_{j}\right) \exp \left(\mathrm{i} \bar{\varphi}_{j}\right),
$$

$r_{j}$ being the squeeze parameter and $\bar{\varphi}_{j}$ the squeeze phase. If $\bar{n}_{j}=0$, the initially squeezed state is obtained which reduces to the coherent state provided that $r_{j}=0$ $\left(B_{j}=1, C_{j}=0\right)$. 


\section{Results and discussion}

Using the above expressions the single-mode and compound mode cases can be discussed from the point of view of photon statistics in the same way as in [3]. All relevant equations to calculate the photon number distributions $p(n, t)$, its factorial moments $\left\langle W^{k}(t)\right\rangle$, quadrature variances $\left\langle(\Delta \hat{q}(t))^{2}\right\rangle$ and $\left\langle(\Delta \hat{p}(t))^{2}\right\rangle$ and the principal squeeze parameter $\lambda(t)$ [10] have been given in section 2.1 and 2.2 of the paper [3] for single mode and compound mode cases, respectively. We mainly provide results in the form of reduced factorial moments for the compound modes involving the coupling of modes, i.e. we use the following quantities

$$
\begin{gathered}
F_{i j}^{(k)}=\frac{\left\langle W_{i j}^{k}\right\rangle}{\left\langle W_{i j}\right\rangle^{k}}-1, \quad W_{i j}=W_{i}+W_{j}, \\
F_{i j}^{(2)}=\frac{\left\langle\left(\Delta W_{i j}\right)^{2}\right\rangle}{\left\langle W_{i j}\right\rangle^{2}}, \quad\left(\Delta W_{i j}\right)^{2}=\left(\Delta W_{i}\right)^{2}+\left(\Delta W_{j}\right)^{2}+2 \Delta W_{i} \Delta W_{j}, \\
i, j=\mathrm{S}, \mathrm{A}, \mathrm{V}, \quad i \neq j,
\end{gathered}
$$

$W$ denotes the integrated intensity. For the principal squeeze parameter we have

$$
\lambda(t)=-1+B-|C|,
$$

where $B=B_{i}+B_{j}-2 \operatorname{Re} \bar{D}_{i j}$ and $C=C_{i}+C_{j}+2 D_{i j}$ (the compound quadrature components are chosen as $\left(\hat{A}_{i}+\hat{A}_{i}^{\dagger}+\hat{A}_{j}+\hat{A}_{j}^{\dagger}\right) / \sqrt{2}$ and $\left(\hat{A}_{i}-\hat{A}_{i}^{\dagger}+\hat{A}_{j}-\hat{A}_{j}^{\dagger}\right) / \sqrt{2} \mathrm{i}$, $i \neq j)$.

The following initial phase relations of phases $\varphi_{j}$ of the initial complex amplitudes $\xi_{j}$ have been considered:

(i) $\Delta \varphi=\varphi_{\mathrm{S}}+\varphi_{\mathrm{A}}-2 \varphi_{\mathrm{L}}=0$,

(ii) $\Delta \varphi=\pi$,

(iii) $\Delta \varphi=-\pi$.

The phases of rotating terms $\left(C_{j}\right)$ are $\bar{\varphi}_{\mathrm{S}}=\bar{\varphi}_{\mathrm{A}}=\bar{\varphi}_{\mathrm{V}}=\pi$, whereas the phases of coupling constants $\operatorname{are} \arg g_{\mathrm{S}}=\arg g_{\mathrm{A}}=\pi / 2$; further we have chosen the values $\left|g_{\mathrm{s}}\right|=10^{7} \mathrm{~s}^{-1},\left|g_{\mathrm{A}}\right|=2 \times 10^{7} \mathrm{~s}^{-1}$ for the coupling constants, $\gamma=$ $10^{8} \mathrm{~s}^{-1}$ for the damping constant (if not given othervise) and $\bar{n}_{j}=\left\langle n_{\mathrm{d} j}\right\rangle=0$. In order to obtain the maximum in nonclassical behaviour, the value of the squeeze parameter $r_{j} \approx 0.37$ was found to be appropriate. In the following figures we note $\cdot\left(\left|g_{\mathrm{A}}\right|^{2}-\left|g_{\mathrm{S}}\right|^{2}\right)^{\frac{1}{2}} t=\Omega t$ simply as $t$.

In Fig. 1 we see the second reduced factorial moment $F_{\mathrm{SA}}^{(2)}$ for $r_{\mathrm{V}}=0$ (unsqueezed phonons) under the phase conditions (3.2). Below zero we have sub-Poisson behaviour. We see that the effects return periodically provided that losses are neglected. The phase condition (i) can lead to super-chaotic (super-Gaussian) statistics, whereas the conditions (ii) and (iii) admit only small sub-chaotic fluctuations.

The time evolution of $F_{\mathrm{SA}}^{(k)}, k=2-5$ together with the mean integrated intensity $\left\langle W_{\mathrm{SA}}\right)$ is shown in Fig. 2 for initially sub-Poisson light $(\Delta \varphi=-\pi$, the other parameters are as in Fig. 1). One can observe that attenuation of the resulting 


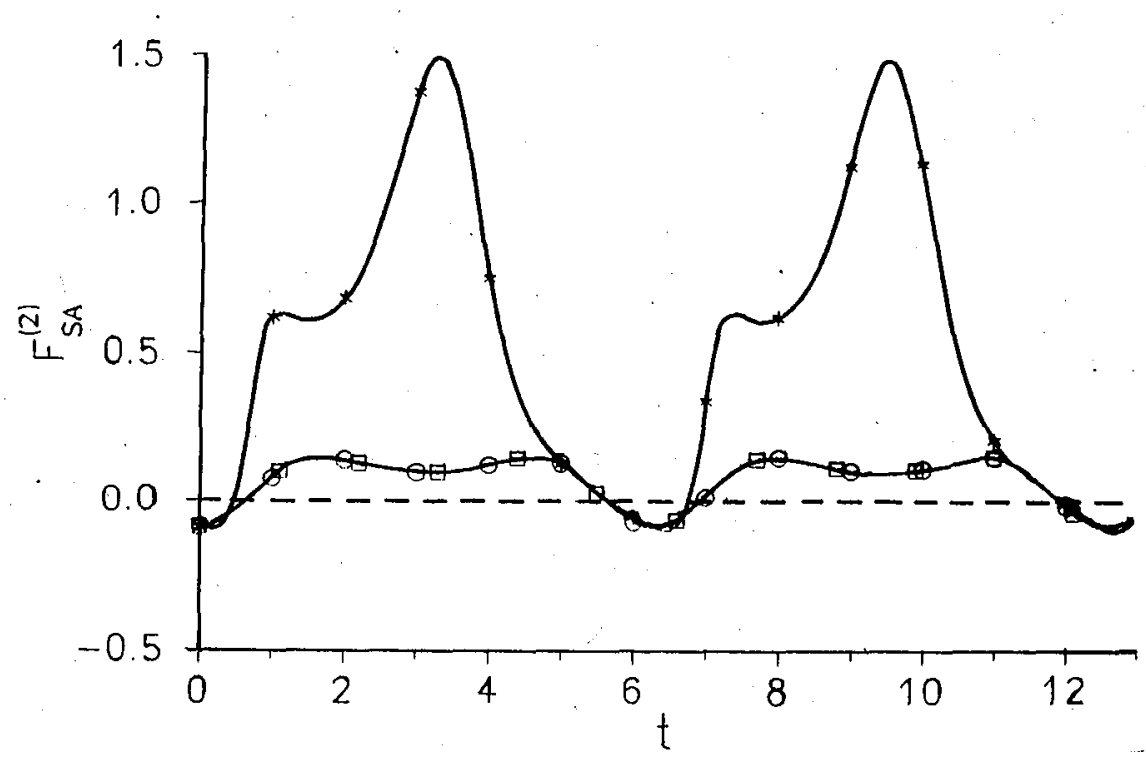

.Fig. 1. Time evolution of the second reduced factorial moment in the compound mode $\mathrm{SA}, 2\left|g_{\mathrm{S}}\right|=\left|g_{\mathrm{A}}\right|=2 \times 10^{7} \mathrm{~s}^{-1},\left|\xi_{\mathrm{S}}\right|^{2}=\left|\xi_{\mathrm{A}}\right|^{2}=\left|\xi_{\mathrm{V}}\right|^{2}=2, \gamma=0 \mathrm{~s}^{-1}, r_{\mathrm{S}}=r_{\mathrm{A}}=0.37$, $r_{\mathrm{V}}=0$ for $\Delta \varphi=0(\star), \Delta \varphi=\pi(0)$ and $\Delta \varphi=-\pi(\square)$.

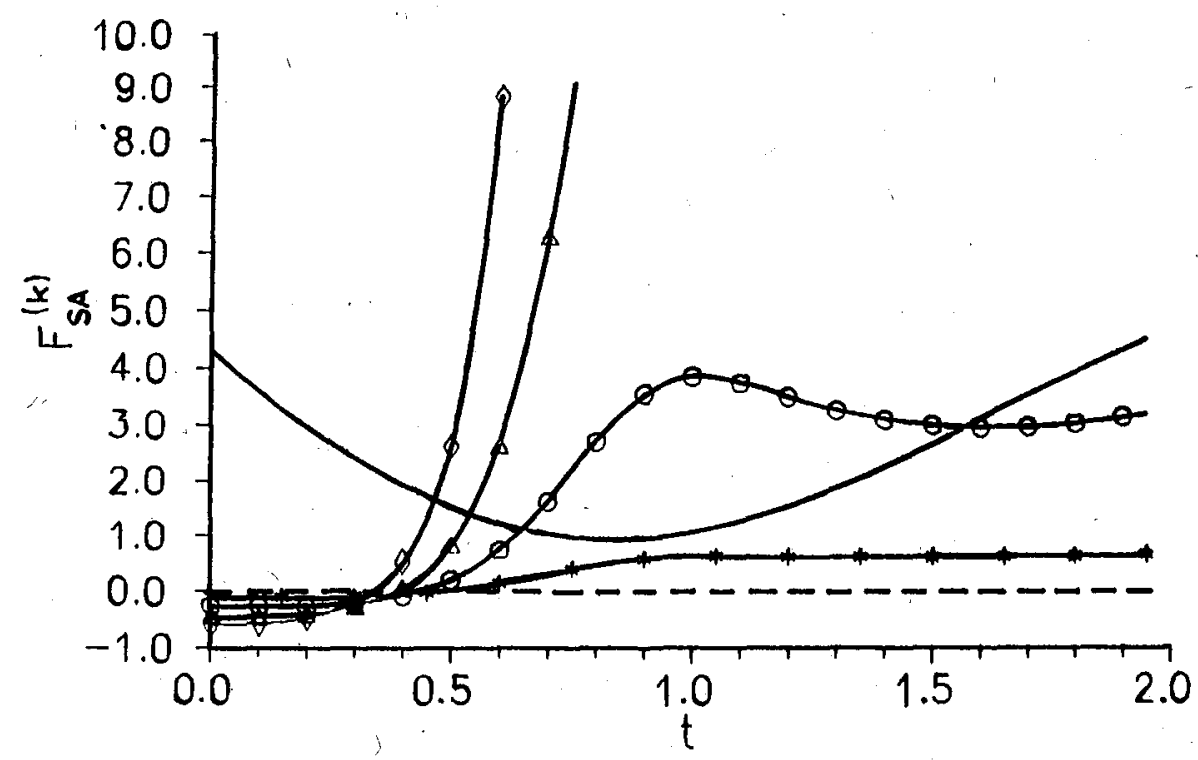

Fig. 2. Time evolution of the reduced factorial moments $F_{\mathrm{SA}}^{(k)}, k=2(\star), k=3$ (0), $k=4(\triangle)$ and $k=5(\diamond)$ and of the resulting intensity $\left\langle W_{\mathrm{SA}}\right\rangle$ (full curve without marks) for $\Delta \varphi=-\pi$, the other parameters are as in Fig. 1. 
integrated intensity is related to a conservation of nonclassical behaviour, whereas in the region where the attenuation is reversed to the amplification, there is a strong increase of quantum noise, which is reduced again for later times.

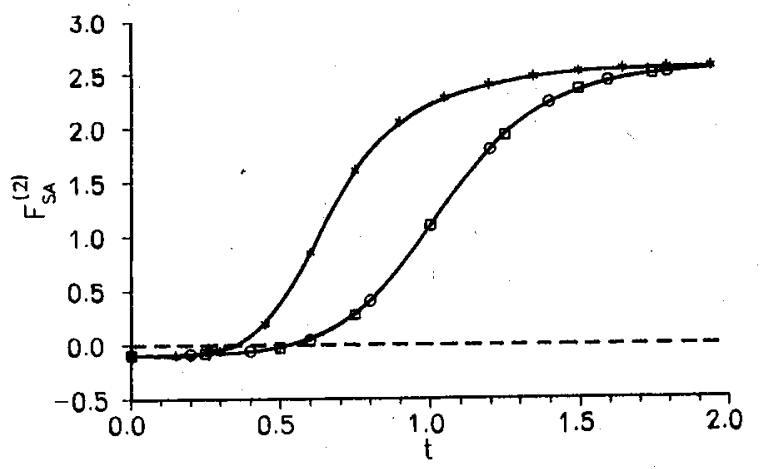

Fig. 3. As in Fig. 1 for $\gamma=10^{8} \mathrm{~s}^{-1}$.

If the system is damped $\left(\gamma=10^{8} \mathrm{~s}^{-1}\right)$, one can see in Fig. 3 that relatively long-time conservation of the initial sub-Poisson behaviour is reached for the considered initial phase conditions and finally a super-chaotic state is obtained for long interaction times.

In Fig. 4 we demonstrate the time evolution of $F_{i j}^{(2)}$ for combined modes SA, VS and VA if the phonon system is squeezed $\left(r_{\mathrm{V}}=0.37\right)$ under the phase condition (i). We see that the most pronounced nonclassical effects are in photon-phonon

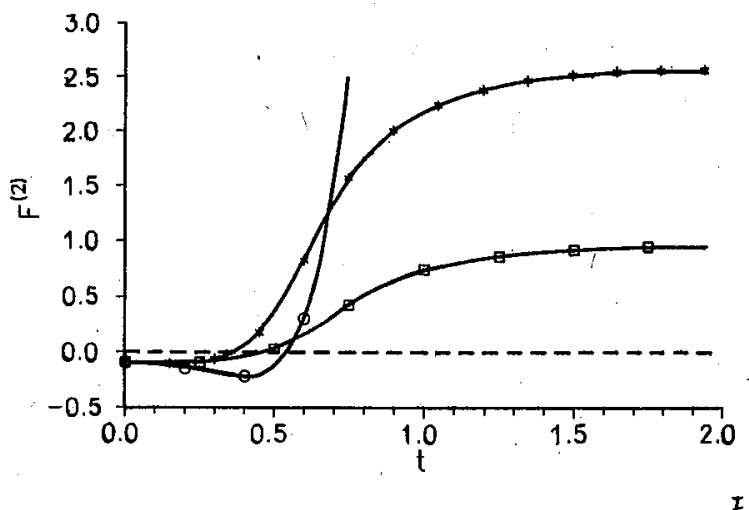

Fig. 4. Time evolution of the second reduced factorial moment for compound modes $\mathrm{SA}(\star), \mathrm{VS}(\circ)$ and $\operatorname{VA}(\square), \Delta \varphi=0, r_{\mathrm{V}}=0.37$, the other parameters as in Fig. 3.

modes and also saturation effects are evident.

As shown in Fig. 5 the correlation properties of the Stokes and anti-Stokes modes are slightly dependent on the squeeze parameter $r_{\mathrm{V}}$ of the phonon system and a super-chaotic state is asymptotically reached. 


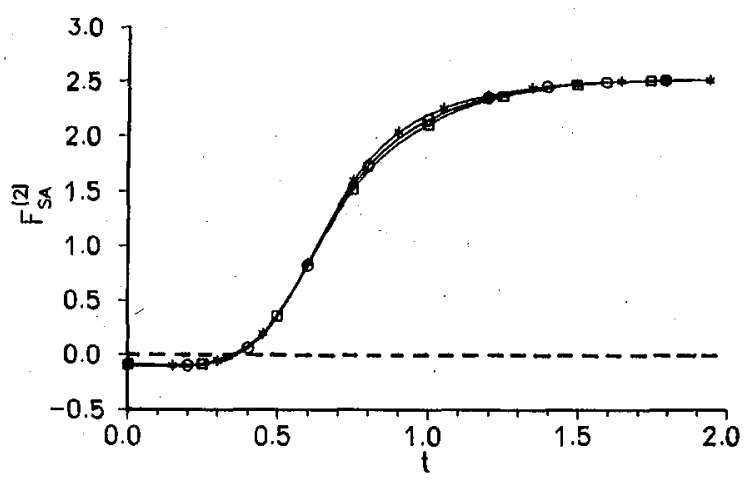

Fig. 5. As in Fig. 3 for $\Delta \varphi=0, r_{\mathrm{V}}=0(\star), r_{\mathrm{V}}=0.37(0)$ and $r_{\mathrm{V}}=0.5$ ().

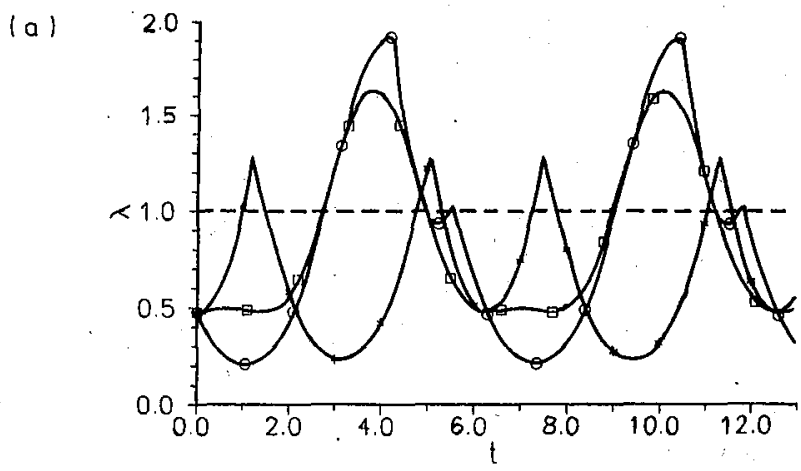

(b)

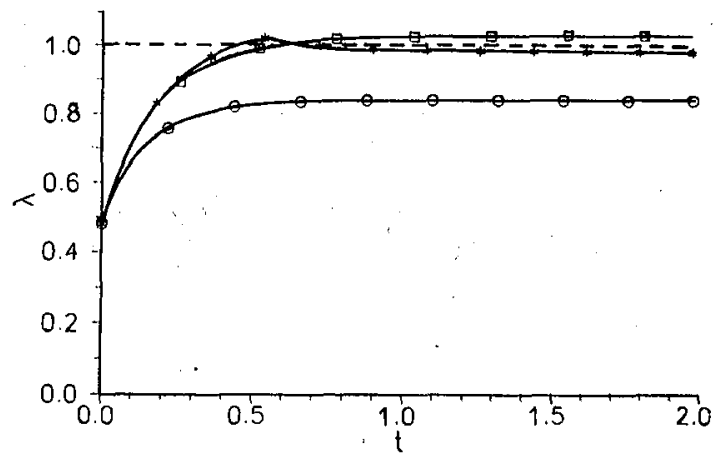

Fig. 6. Time evolution of the principal squeeze parameter $\lambda, \Delta \varphi=0, r_{\mathrm{V}}=0.37$ for mode SA ( $\star$ ), VS (o) and VA (ㅁ) for a) $\gamma=0, b) \gamma=10^{8} \mathrm{~s}^{-1}$; squeezing occurs for the values below 1 . 
(a)

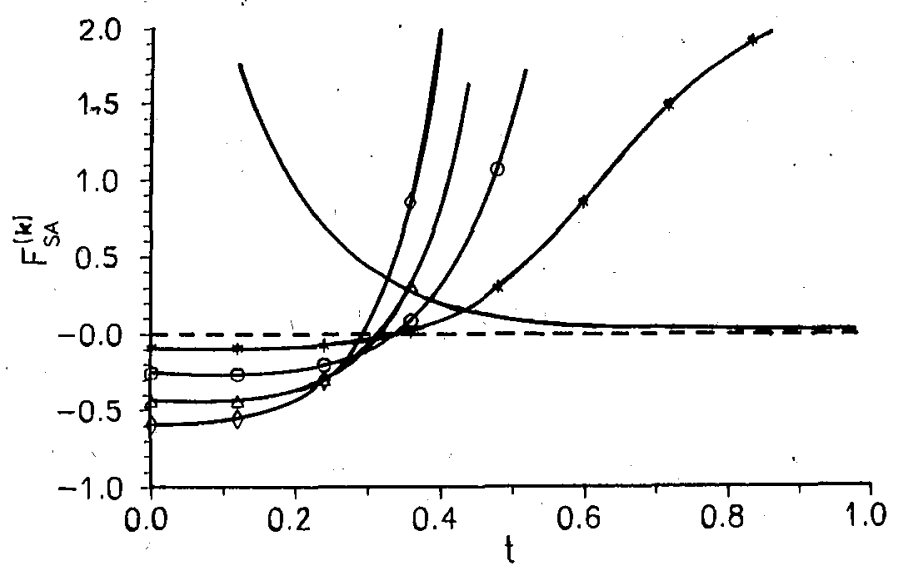

(b)

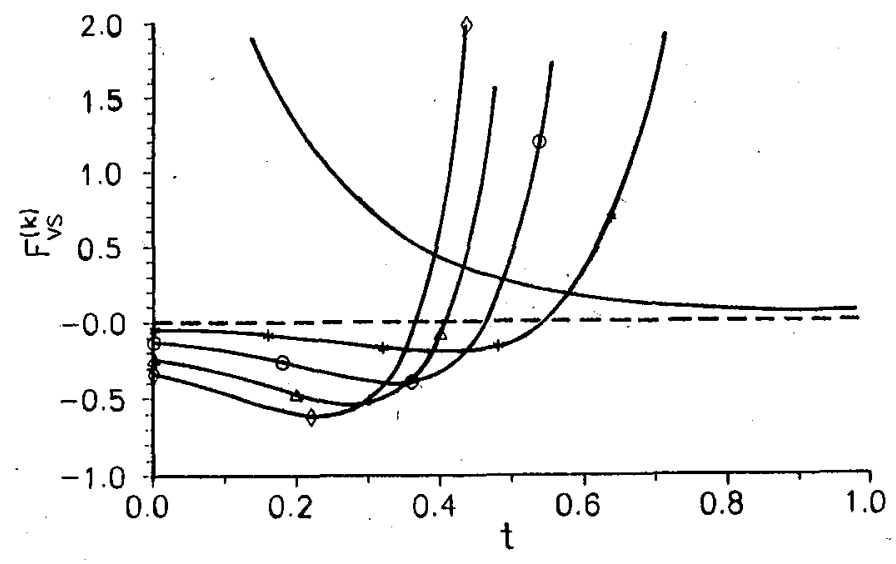

(c)

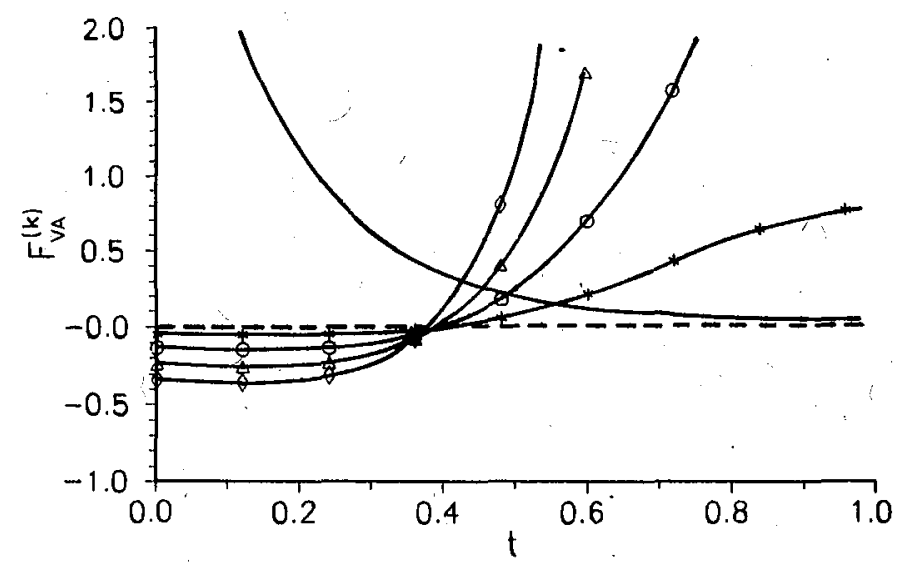

Fig. 7. Time evolution of the reduced factorial moments of the order $k=2(\star), k=3$ $(0), k=4(\Delta)$ and $k=5(\diamond)$ for a) mode $\mathrm{SA}, \mathrm{b})$ mode VS and c) mode VA; $r_{\mathrm{V}}=0$, $\Delta \varphi=0$ and the other parameters as in Fig. $3 ;\langle W\rangle$ is shown by full curve without marks. 


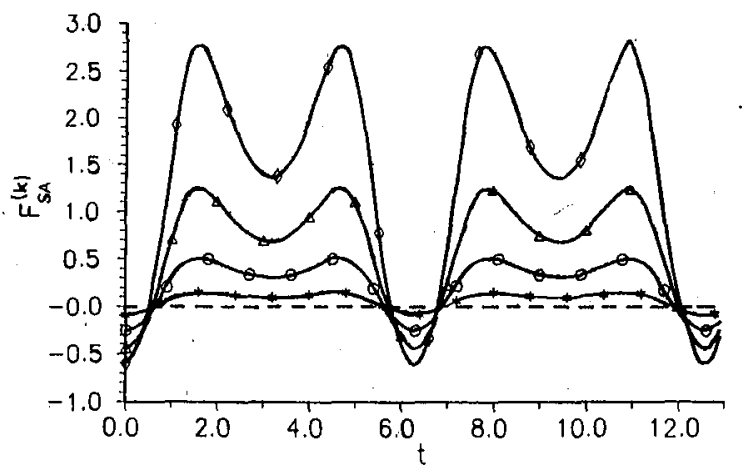

Fig. 8. As in Fig. 2 for the SA mode.

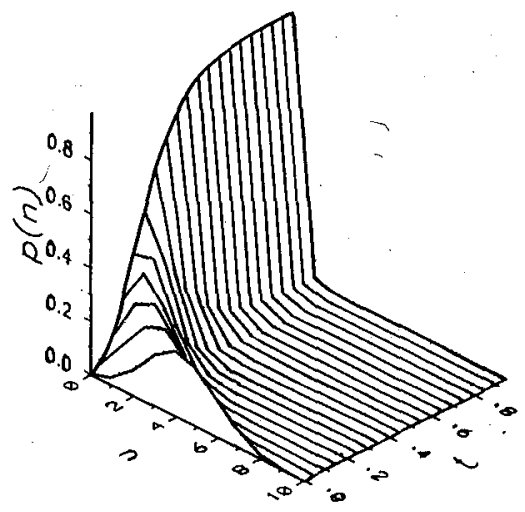

Fig. 9. Time evolution of photon number distribution for the scattered radiation mode $\mathrm{SA}, \Delta \varphi=0, r_{\mathrm{V}}=0$, the other parameters as in Fig. 3 .

In Fig. 6 we demonstrate results in light scattering on squeezed phonons exposing the evolution of the principal squeeze parameter $\lambda$ without and with damping. We see that substantial squeezing of vacuum fluctuations can be obtained in all combined modes (values below $\lambda=1$ ) including the effect of nonlinear dynamics. In modes SA and VS stationary nonclassical effects can happen despite of the damping is reckoned.

In Fig. 7 we demonstrate the reduced factorial moments $F_{i j}^{(k)}, k=2-5$ and the integrated intensity $\langle W\rangle$ in modes SA (Fig. 7 a), VS (Fig. 7 b) and VA (Fig. 7 c) for unsqueezed phonons $\left(r_{V}=0\right)$ and $\Delta \varphi=0$ with the other parameters as in Fig. 3. These figures demonstrate that under the chosen conditions the initial sub-Poisson behaviour can be improved in the photon-phonon modes by the nonlinear dynamics, which supports nonclassical behaviour in photon-phonon correlations more effectively than in photon-photon correlations. 

glected.

The sub-Poisson behaviour can periodically return (Fig. 8) if losses are ne-

Figure 9 provides the time evolution of the photon number distribution for the scattered compound mode SA under the phase matching $(\Delta \varphi=0)$ and for unsqueezed phonon system $\left(r_{\mathrm{V}}=0\right)$ demonstrating the evolution from the initially sub-Poisson statistics to strongly super-chaotic statistics.

Finally we note that the external noise $\bar{n}_{j}$ and reservoir noise $\left\langle n_{\mathrm{d} j}\right\rangle$ generally lead to the degradation of nonclassical behaviour of light.

\section{References}

[1] A. Pieczonková, J. Peřina, Czech. J. Phys. B 31, 837 (1981).

[2] A. Pieczonková, Czech. J. Phys. B 32, 831 (1982); Opt. Acta 29, 1509 (1982).

[3]. M. Kárská, J. Peřina, J. Mod. Opt. 37, 195 (1990) and reférences therein.

[4] P. Král, Czech. J. Phys. B 40, 1226 (1990).

[5] M.C. Raymer, I.A. Walmsley, Progress in Optics, Vol. 28, ed. E. Wolf, North-Holland, Amsterdam 1990.

[6] J. Peřina, Quantum Statistics of Linear and Nonlinear Optical Phenomena, D. Reidel, Dordrecht-Boston 1984 (2nd enlarged edition 1991).

[7] J. Peřina, J. Bajer, J. Křepelka, Z. Hradil, J. Mod. Opt. 34, 965 (1987).

[8] V. Peřinová, J. Křepelka, Phys. Rev. A 39, 4045 (1989).

[9] J. Bajer, Czech. J. Phys. B 40, 646 (1990).

[10] A. Luks̃, V. Per̆inová, J. Per̆ina, Opt. Commun. 67, 149 (1988). 